\title{
Fluoro-deoxyglucose-positron emission tomography/CT scan in superior vena cava syndrome due to radiation-induced fibrosis
}

\author{
Katsunori Kagohashi, Hiroaki Satoh \\ Division of Respiratory Medicine, Mito Medical Center, University of Tsukuba, Mito-city, Japan
}

Key words: fluoro-deoxyglucose-positron emission tomography; CT scan: superior vena cava syndrome; lung cancer

Rep Pract Oncol Radiother 2021;26(3):451-453

A 43-year-old man with a 20 pack-year history of smoking presented with chest pain and hemoptysis that began one month previously. Chest computed tomography (CT) confirmed a $5-\mathrm{cm}$ mass in the right upper lobe involving the right hilum with occlusion of the right main stem bronchus with ipsilateral mediastinal lymph node enlargement (Fig. 1A). The primary tumor extended into the mediastinum, but there was no external compression of the carina. The patient was diagnosed as having stage IIIB non-small cell lung cancer. He received concurrent chemo-radiotherapy with cisplatin and etoposide and $60 \mathrm{~Gy}$ of radiation to the primary tumor. The dose distribution was calculated based on the CT image, and three-dimensional irradiation was performed. However, the tumor invaded the superior vena cava (SVC); hence, irradiation of the SVC was unavoidable. Fluoro-deoxyglucose-positron emission tomography (FDG-PET)/ /CT scan performed three months after treatment completion demonstrated complete response to the chemo-radiotherapy. FDG-PET/CT also showed no uptake at a radiation fibrosis near the SVC. At 48 months of follow-up, the patient presented with progressive facial and neck swelling. FDG-PET/CT scan showed pinpoint narrowing of the SVC. As the radiation fibrotic lesion near the SVC did not enlarge or present FDG uptake (Fig. 1B), SVC syndrome was evaluated not by recurrence of lung cancer but by the fibrotic lesion. Stent placement in the SVC and anticoagulation with warfarin was initiated. SVC syndrome was controlled by replacement and development of collateral circulation. Since pleural disseminated lesions were found in the CT scan at that time, the disseminated lesions were treated with irradiation, subsequent chemotherapy and immune checkpoint inhibitors. Thereafter, we conducted FDG-PET/CT scans six times every six months, but the radiation fibrotic lesion near the SVC did not enlarge or present FDG uptake. Two years after the onset of SVC syndrome, the lesion near the SVC had not enlarged and presented no FDG uptake in the FDG-PET/CT scan (Fig. 1C). The patient died of meningeal carcinomatosis that developed 7 years after the initial chemo-radiotherapy. Autopsy examination was not performed.

The majority of patients with SVC syndrome are those with cancer-related SVC syndrome [1]. Embolism associated with a tumor should also be included in the differential diagnosis of SVC syndrome in cancer patients [1]. Radiation fibrotic lesions have also been attributed to the activation and proliferation of fibroblasts that secrete unorganized extracellular matrix. Transforming growth 

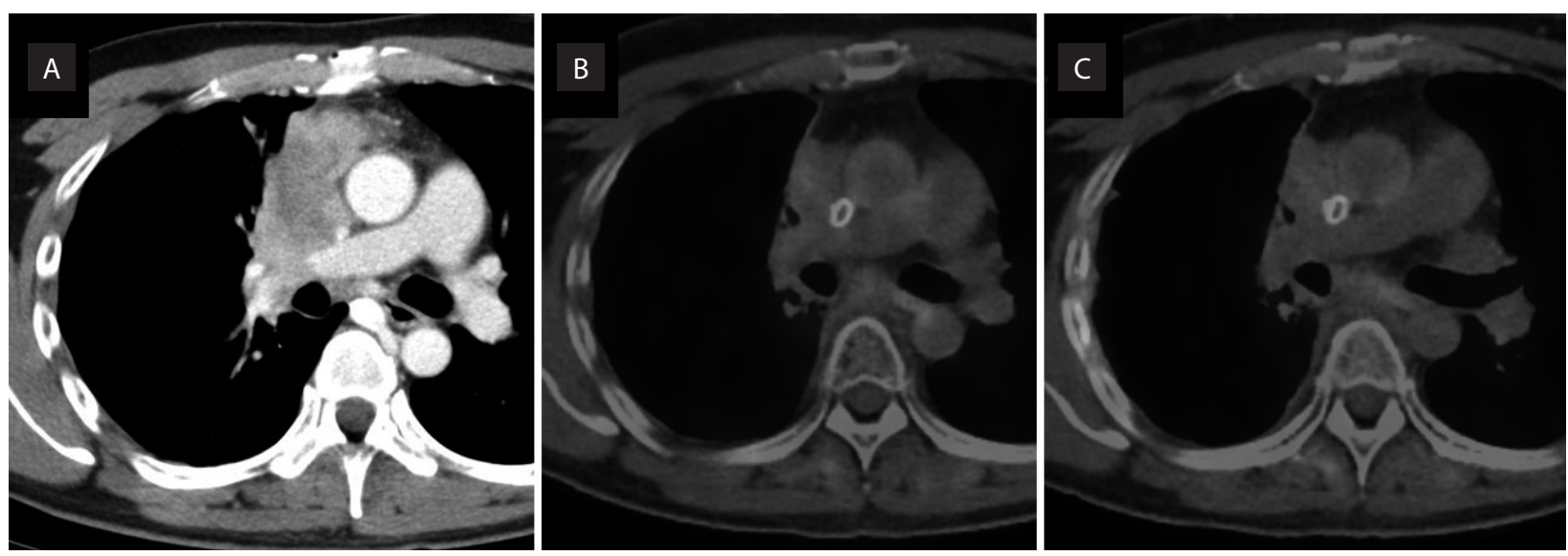

Figure 1. Chest computed tomography (CT) at admission showed an enlargement of mediastinal lymph node on the right (A). Fluoro-deoxyglucose-positron emission tomography (FDG-PET)/CT scan taken at the time of development of superior vena cava syndrome which showed no enlargement or uptake at the radiation fibrotic lesion near the superior vena cava (B). FGD-PET/CT scan taken two years after the development of superior vena cava (SVC) syndrome. The lesion near the SVC did not enlarge or had no FDG uptake (C)

factor-beta (TGF- $\beta$ ) is evaluated as one of the key cytokines for the initiation as well as proliferation of radiation fibrotic lesions [3]. TGF- $\beta$ leads to the recruitment and activation of fibroblasts. Its release by platelets and myoblasts after irradiation leads to a vicious cycle of fibrosis [2]. Patients with SVC syndrome due to fibrotic lesions formed by irradiation have been very rare [4-9]. A PubMed search revealed six such patients [4-9]. Two of them developed SVC syndrome after chemoradiotherapy $[8,9]$. FDG-PET/CT was performed in two of the other four patients. In them, FDG uptake was not observed in the radiation fibrotic lesion formed at the irradiation site. That result was consistent with ours. The follow-up period for these two patients was nine months and one year after the onset of SVC syndrome, respectively. Our patient was followed up for five years, during which several FDG-PET examinations confirmed that there was no FDG uptake in the radiation fibrotic lesion. None of the other described patients had several PDG-PET imaging tests as our patient had. The follow-up period of the four patients since onset of SVC syndrome was between six months and three years. In our patient, the follow-up period since onset of SVC syndrome was five years. Five of the encountered patients had information available regarding their treatment for SVC syndrome. Among them, three patients had surgical bypass grafting of the SVC, one patient had a stent inserted, and one patient received only anticoagulation with warfarin.
SVC surgical bypass transplant is often performed for SVC syndrome due to non-malignant causes because of a lower rate of re-occlusion in these patients [10]. Our patient underwent stenting and was given warfarin but did not develop thromboembolism. His treatment was determined and implemented in consideration of the patient's general condition, local and systemic cancer control status, and life expectancy.

In conclusion, in patients with SVC in the irradiation field, an FDG-PET/CT scan might be useful to determine whether the SVC syndrome is due to cancer or non-cancerous lesions.

\section{Conflict of interest}

None declared.

\section{Funding}

None declared.

\section{Authors' contributions}

K.K., H.S. designed the study, collected the data, analyzed the data, and prepared the manuscript. Both authors approved the final version for submission.

\section{Ethics}

Written comprehensive consent was obtained from patient. Reporting of this case report was approved by the Ethics Committee in Mito Medical Center, University of Tsukuba Hospital (NO 16-66). 


\section{References}

1. Yellin A, Rosen A, Reichert N, et al. Superior vena cava syndrome. The myth--the facts. Am Rev Respir Dis. 1990; 141 (5 Pt 1): 1114-1118, doi: 10.1164/ajrccm/141.5_Pt_1.1114, indexed in Pubmed: 2339833.

2. Delanian S, Lefaix JL. The radiation-induced fibroatrophic process: therapeutic perspective via the antioxidant pathway. Radiother Oncol. 2004; 73(2): 119-131, doi: 10.1016/j. radonc.2004.08.021, indexed in Pubmed: 15542158.

3. Yarnold J, Brotons MCV. Pathogenetic mechanisms in radiation fibrosis. Radiother Oncol. 2010; 97(1): 149-161, doi: 10.1016/j.radonc.2010.09.002, indexed in Pubmed: 20888056.

4. Van Putten JW, Schlosser NJ, Vujaskovic Z, et al. Superior vena cava obstruction caused by radiation induced venous fibrosis. Thorax. 2000; 55(3): 245-246, doi: 10.1136/ thorax.55.3.245, indexed in Pubmed: 10679546.

5. Lee $Y$, Doering $R$, Jihayel $A$. Radiation-induced superior vena cava syndrome. Tex Heart Inst J. 1995; 22(1): 103-104, indexed in Pubmed: 7787461.
6. Dhaliwal RS, Das D, Luthra S, et al. Management of superior vena cava syndrome by internal jugular to femoral vein bypass. Ann Thorac Surg. 2006; 82(1): 310-312, doi: 10.1016/j.athoracsur.2005.08.059, indexed in Pubmed: 16798237.

7. Mehta SV, Koo DJ. Radiation-induced SVC syndrome. BMJ Case Rep. 2014; 2014, doi: 10.1136/bcr-2013-203446, indexed in Pubmed: 24554686.

8. Castonguay M, Rodrigues $G$, Vincent $M$, et al. Chemoradiation-induced superior vena cava syndrome: a case report. Can Respir J. 2008; 15(8): 444-446, doi: 10.1155/2008/279786, indexed in Pubmed: 19107246.

9. Hinton J, Cerra-Franco A, Shiue K, et al. Superior vena cava syndrome in a patient with locally advanced lung cancer with good response to definitive chemoradiation: a case report. J Med Case Rep. 2018; 12(1): 301, doi: 10.1186/ s13256-018-1843-4, indexed in Pubmed: 30340621.

10. Kalra M, Gloviczki P, Andrews J, et al. Open surgical and endovascular treatment of superior vena cava syndrome caused by nonmalignant disease. JVasc Surg. 2003; 38(2): 215-223, doi: 10.1016/s0741-5214(03)00331-8, indexed in Pubmed: 12891100. 